7. R. L. Duncan. A variation of the Buffon needle problem. this Magazine, 40 (1967), 36-38.

8. H. J. Khamis. Buffon's needle problem on radial lines. this MAGAZINE, 64 (1991), 56-58.

9. Daniel A. Klain and Gian-Carlo Rota. Introduction to geometric probability. Lezioni Lincee. [Lincei Lectures]. Cambridge University Press, Cambridge, 1997.

10. Pierre Simon Laplace. Théorie Analytique des Probabilités. 1812.

11. Mario Lazzarini. Un' applicazione del calcolo della probabilità alla ricerca sperimentale di un valore approssimato di $\pi$. Periodico di Matemtica, 4 (1901), 140-143.

12. M. F. Neuts and P. Purdue. Buffon in the round. this Magazine, 44 (1971), 81-89.

13. J. F. Ramaley. Buffon's noodle problem. Amer. Math. Monthly, 76 (1969), 916-918.

14. J. V. Uspensky. Introduction to Mathematical Probability. McGraw Hill Book Company, Inc., New York, 1937.

\title{
Conditions Equivalent to the Existence of Odd Perfect Numbers
}

\author{
JUDY A. HOLDENER \\ Kenyon College \\ Gambier, OH 43022 \\ holdenerj@kenyon.edu
}

The abundancy index $I(n)$ of a positive integer $n$ is defined to be the ratio $I(n)=$ $\sigma(n) / n$, where $\sigma(n)=\sum_{d \mid n} d$. This index is a useful tool in determining whether a number is deficient, abundant, or perfect. In particular, $n$ is deficient if $I(n)<2$, it is abundant if $I(n)>2$, and perfect if $I(n)=2$. Some of the oldest open problems in mathematics relate to the abundancy of a number. Are there infinitely many perfect numbers? Does there exist an odd perfect number? Here are just two questions that were posed by the Greeks over two thousand years ago, and yet they remain unanswered today.

In recent years, this MAGAZINE has published several interesting articles examining the abundancy index of a number $[\mathbf{1}, \mathbf{3}, \mathbf{4}]$. In $[\mathbf{1}]$, R. Laatsch provided a comprehensive summary of what is known about the abundancy index, including a proof that the image of $I(n)$ is dense in the interval $(1, \infty)$. He also posed several interesting questions, one of which was: Is every rational number $q>1$ the abundancy index of some integer? In [4], P. A. Weiner answered Laatsch's question in the negative by providing an infinite family of rational numbers in $(1, \infty)$ which fail to be an abundancy index of any integer. Even more interesting, Weiner proved that the set of rationals in $(1, \infty)$ not in the range of $I(n)$ is actually dense in $(1, \infty)$. Finally, Weiner proved the following result:

THEOREM. (WEINER, 2000) If I $(n)=\frac{5}{3}$ for some $n$, then $5 n$ is an odd perfect number.

In [3], R. F. Ryan then generalized this theorem of Weiner by proving the following:

THEOREM. (RYAN, 2003) If there exists a positive integer $n$ and an odd positive integer $m$ such that $2 m-1$ is a prime not dividing $n$ and

$$
I(n)=\frac{2 m-1}{m},
$$

then $n(2 m-1)$ is an odd perfect number.

In Theorem 1 below, we generalize Ryan's result further by providing a condition that is actually equivalent to the existence of odd perfect numbers. The generalization 
is also helpful in that it sheds some light on the theorems cited above by revealing the connection that they have to Euler's well-known characterization of odd perfect numbers. (Euler proved that if an odd perfect number exists, it must have the form $p^{\alpha} m^{2}$, where $p$ is a prime satisfying $\operatorname{gcd}(p, m)=1$ and $p \equiv \alpha \equiv 1(\bmod 4)[2$, p. 267].)

THEOREM 1. There exists an odd perfect number if and only if there exist positive integers $p, n$, and $\alpha$ such that $p \equiv \alpha \equiv 1(\bmod 4)$, where $p$ is a prime not dividing $n$, and

$$
I(n)=\frac{2 p^{\alpha}(p-1)}{p^{\alpha+1}-1} .
$$

Proof. If $N$ is an odd perfect number, then Euler showed that $N$ must have the form $N=p^{\alpha} m^{2}$, where $p$ is a prime satisfying $\operatorname{gcd}(p, m)=1$ and $p \equiv \alpha \equiv 1(\bmod 4)$. Hence $\sigma(N)=\sigma\left(p^{\alpha} m^{2}\right)=\sigma\left(p^{\alpha}\right) \sigma\left(m^{2}\right)=2 p^{\alpha} m^{2}$, and

$$
I\left(m^{2}\right)=\frac{\sigma\left(m^{2}\right)}{m^{2}}=\frac{2 p^{\alpha}}{\sigma\left(p^{\alpha}\right)}=\frac{2 p^{\alpha}(p-1)}{p^{\alpha+1}-1} .
$$

This proves the forward direction of the theorem.

Conversely, assume that there exists a positive integer $n$ such that

$$
I(n)=\frac{2 p^{\alpha}(p-1)}{p^{\alpha+1}-1},
$$

where $p \equiv \alpha \equiv 1(\bmod 4)$ and $p$ is a prime satisfying $p \nmid n$. Then

$$
I\left(n \cdot p^{\alpha}\right)=I(n) I\left(p^{\alpha}\right)=\frac{2 p^{\alpha}(p-1)}{p^{\alpha+1}-1} \cdot \frac{p^{\alpha+1}-1}{p^{\alpha}(p-1)}=2 .
$$

So $n \cdot p^{\alpha}$ is a perfect number.

Now we claim that $n \cdot p^{\alpha}$ cannot be even. For if it were, it would have the EuclidEuler form for even perfect numbers:

$$
n \cdot p^{\alpha}=2^{m-1}\left(2^{m}-1\right)
$$

where $2^{m}-1$ is prime. Since $2^{m}-1$ is the only odd prime factor on the right hand side, $p^{\alpha}=p^{1}=2^{m}-1$. But $p \equiv 1(\bmod 4)$ and $2^{m}-1 \equiv 3(\bmod 4)$ (because $m$ must be at least 2 in order for $2^{m}-1$ to be prime). This contradiction shows that $n \cdot p^{\alpha}$ is not even. Therefore it is an odd perfect number.

In closing, we note that if $I(n)=5 / 3$ then 5 cannot be a divisor of $n$. (See the proof of Theorem 3 in [4].) Hence Theorem 1 yields Weiner's result when $p=5$ and $\alpha=1$. If $\alpha=1$ and $p=4 k+1$, then

$$
\frac{2 p^{\alpha}(p-1)}{p^{\alpha+1}-1}=\frac{2 p}{p+1}=\frac{2(4 k+1)}{4 k+2}=\frac{2(2 k+1)-1}{2 k+1},
$$

and by setting $m=2 k+1$, we see that Theorem 1 generalizes Ryan's result as well.

Acknowledgment. I would like to thank Paul Weiner who suggested the simpler proof of the converse to Theorem 1 appearing here. Thanks, too, to an anonymous referee who offered valuable feedback on this work and to the Department of Mathematics at the University of Colorado in Boulder (and David Grant, in particular) for the support they provided me during the writing of this paper. 


\title{
REFERENCES
}

1. R. Laatsch, Measuring the abundance of integers, this MAGAZINE 59 (1986), 84-92.

2. K. H. Rosen, Elementary Number Theory and its Applications, 5th ed., Pearson Addison Wesley, Boston, 2005.

3. R. F. Ryan, A simpler dense proof regarding the abundancy index, this MAGAZINE 76 (2003), 299-301.

4. P. A. Weiner, The abundancy index, a measure of perfection, this MAgazine 73 (2000), 307-310.

\section{The Associativity of the Symmetric Difference}

\author{
MAJID HOSSEINI \\ State University of New York at New Paltz \\ New Paltz, NY 12561-2443 \\ hosseinm@newpaltz.edu
}

The symmetric difference of two sets $A$ and $B$ is defined by $A \Delta B=(A \backslash B) \cup$ $(B \backslash A)$. It is easy to verify that $\Delta$ is commutative. However, associativity of $\Delta$ is not as straightforward to establish, and usually it is given as a challenging exercise to students learning set operations (see [1, p. 32, exercise 15], [3, p. 34, exercise 2(a)], and [2, p. 18]).

In this note we provide a short proof of the associativity of $\Delta$. This proof is not new. A slightly different version appears in Yousefnia [4]. However, the proof is not readily accessible to anyone unfamiliar with Persian.

Consider three sets $A, B$, and $C$. We define our universe to be $X=A \cup B \cup C$. For any subset $U$ of $X$, define the characteristic function of $U$ by

$$
\chi_{U}(x)= \begin{cases}1, & \text { if } x \in U ; \\ 0, & \text { if } x \in X \backslash U .\end{cases}
$$

Two subsets $U$ and $V$ of $X$ are equal if and only if $\chi_{U}=\chi_{V}$. The following lemma is the key to our proof.

Lemma. For any two subsets $U$ and $V$ of $X$ and for any $x \in X$,

$$
\begin{aligned}
\chi_{U \Delta V}(x) & =\left(\chi_{U}(x)-\chi_{V}(x)\right)^{2} \\
& =\chi_{U}(x)+\chi_{V}(x)-2 \chi_{U}(x) \chi_{V}(x) .
\end{aligned}
$$

Proof. Note that both sides of (1) are equal to 1 exactly when $x$ belongs to one of $U$ or $V$, but not to both. The identity (2) follows immediately from (1) and the fact that $\chi_{S}^{2}=\chi_{S}$ for any set $S$.

Proposition. Let $A, B$, and $C$ be three sets. Then

$$
(A \Delta B) \Delta C=A \Delta(B \Delta C) .
$$

Proof. From the Lemma we see that

$$
\begin{aligned}
\chi_{(A \Delta B) \Delta C} & =\chi_{A \Delta B}+\chi_{C}-2 \chi_{A \Delta B} \chi_{C} \\
& =\left(\chi_{A}+\chi_{B}-2 \chi_{A} \chi_{B}\right)+\chi_{C}-2\left(\chi_{A}+\chi_{B}-2 \chi_{A} \chi_{B}\right) \chi_{C} \\
& =\chi_{A}+\chi_{B}+\chi_{C}-2 \chi_{A} \chi_{B}-2 \chi_{A} \chi_{C}-2 \chi_{B} \chi_{C}+4 \chi_{A} \chi_{B} \chi_{C} .
\end{aligned}
$$

